Proc SPIE ERS08 Proceedings, Vol 7104, Remote Sensing for Agriculture, Ecosystems, and Hydrology X, Cardiff, Wales, United Kingdom, September 15-19, 2008 (Invited Paper)

\title{
Space-based ornithology - studying bird migration and environmental change in North America
}

\author{
James A. Smith*a , Jill L. Deppe**b \\ ${ }^{a}$ NASA Goddard Space Flight Center, Hydrospheric and Biospheric Sciences Laboratory, Greenbelt, \\ MD, USA 20771; \\ ${ }^{\mathrm{b}}$ University of Maryland Baltimore County, Goddard Earth Sciences Technology Center, Baltimore, \\ MD USA 21250
}

\begin{abstract}
Natural fluctuations in the availability of critical stopover sites coupled with anthropogenic destruction of wetlands, land-use change, and anticipated losses due to climate change present migratory birds with a formidable challenge. Space based technology in concert with bird migration modeling and geographical information analysis yields new opportunities to shed light on the distribution and movement of organisms on the planet and their sensitivity to human disturbances and environmental changes. At the NASA Goddard Space Flight Center, we are creating ecological forecasting tools for science and application users to address the consequences of loss of wetlands, flooding, drought or other natural disasters such as hurricanes on avian biodiversity and bird migration. We use an individual-based bird biophysical migration model, driven by remotely sensed land surface data, climate and hydrologic data, and biological field observations to study migratory bird responses to environmental change in North America. Simulation allows us to study bird migration across multiple scales and can be linked to mechanistic processes describing the time and energy budget states of migrating birds. We illustrate our approach by simulating the spring migration of pectoral sandpipers from the Gulf of Mexico to Alaska. Mean stopover length and trajectory patterns are consistent with field observations.
\end{abstract}

Keywords: Satellite remote sensing, bird migration modeling, habitat suitability analysis, environmental change

\section{INTRODUCTION}

The study of bird migration on a global scale is one of the compelling and challenging problems of modern biology with major implications for human health and conservation biology. Natural fluctuations in the availability of critical stopover sites coupled with anthropogenic destruction of wetlands, land-use changes, and anticipated losses due to climate change present migratory birds with a formidable challenge [1]. Indeed, it is estimated that at least $50 \%$ of shorebird species have experienced population declines over the last decades [2]. Conservation biologists are concerned that continental scale reductions in wetland availability, quality or spacing are negatively impacting these species by eliminating critical stopovers along migratory flyways [3].

Space based technology in concert with bird migration modeling and geographical information analysis offers new tools to shed understanding on the distribution and migration of organisms on the planet and their sensitivity to human disturbances and environmental changes [4] [5]. Advances in remote sensing and numerical weather and climate prediction models provide us with measurements of atmospheric and land surface conditions at varying spatial resolution [6]. These data can be provided in near-real time or are archived for retrospective studies.

We are using individually based bird migration models that incorporate bird biophysical properties, movement behavior, avian energetics and spatially and temporally varying environmental suitability [7] [8]. We use remote sensing to obtain spatially and temporally varying properties of the landscape to estimate potential stopover locations for refueling. In this paper we explore the use of AVHRR Normalized Difference Vegetation Index composite observations [9] together with a high-resolution data set of surface climate [10] derived for studies with the Intergovernmental Panel on Climate Change (http/ www ipcech). We illustrate our approach by simulating the spring migration of pectoral sandpipers in North America using a Lagrangian mesh with latitude, longitude grid cell spacing of 10 minutes by 10 minutes.

*James.A.Smith(anasa.gov; phone $1301614-6020$; fax $1301614-5666$

**Now with Illinois Natural History Survey at the University of Illinois in Urbana-Champaign 
Proc SPIE ERS08 Proceedings, Vol 7104, Remote Sensing for Agriculture, Ecosystems, and Hydrology X, Cardiff, Wales, United Kingdom, September 15-19, 2008 (Invited Paper)

\section{METHODOLOGY}

We simulate the migration trajectories of individual birds, including their positions, velocities, and fuel status using a spatially explicit, biophysical flight model. The model incorporates species-specific morphology and physiology parameters. We drive the model using remotely sensed land surface conditions and the output of numerical weather prediction models. We fly birds in a user-specified Lagrangian grid and run the model at a daily time step to simulate spring or fall migration. We keep track of time spent flying, resting, or re-fueling during en-route migratory stopovers.

We can select various subpopulations of a species for study by varying initial starting locations, inherited behaviors, e.g. flight direction preferences, and other parameters. Here, we choose a subpopulation of pectoral sandpipers that continue their northward spring migration after crossing the Gulf of Mexico. We begin our simulation in April and track the birds towards their Northern tundra breeding grounds in June.

\subsection{Study species}

In this study we focus on the spring migration of Pectoral Sandpipers (Calidris melanotos) in North America, Figure Ia. Pectoral sandpipers breed in the wet arctic tundra of northern Alaska and northwestern Canada and winter in grasslands and wetlands of southern and south-central South America. Pectoral Sandpipers are associated with shallow wetlands and flooded or irrigated grasslands and agricultural fields in the interior regions of North America, where they forage on soil invertebrates by probing their long, slender bills into the soil. Sandpipers use managed wetlands as well as natural wetlands. Our model also should be applicable to other shorebirds, e.g. the Black-necked Silt (Himantopus mexicanus), Figure $1 \mathrm{~b}$, that forage by probing and gleaning in mudflats, lakeshores, and in very shallow waters near shores where it seeks out crustaceans, arthropods mollusks, aquatic invertebrates and small fish.

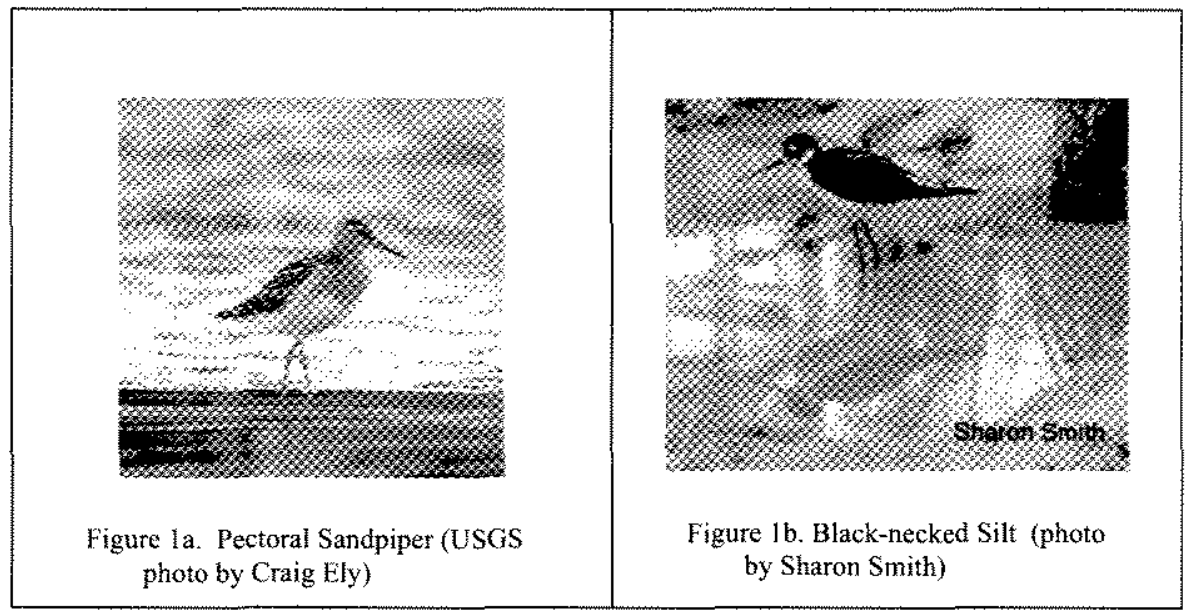

\subsection{Migration Model}

The migration model incorporates various phenotypic behaviors with which we experiment to study the effects of different individual bird flight decision rules on migration success. Iterating over bird populations and running multiple generations allows us to summarize population-level distributions, including arrival and passage dates, residence times distributions and frequency plots of underlying phenotypic characteristics for those birds undergoing successful migration.

By initializing a population with random candidate solutions, repeatedly exposing successive generations to the environment, calculating the fitness of survivors and reproducing and selecting individuals based on their relative fitness for the next generation, we can study the effects of changing environmental conditions on migratory success. For example, what are the impacts of resulting changes in the quality, location, and quantity of stopover habitat, and what is the coupling between the timing of migration and key environmental processes? 
Proc SPIE ERS08 Proceedings, Vol 7104, Remote Sensing for Agriculture, Ecosystems, and Hydrology X, Cardiff, Wales, United Kingdom, September 15-19, 2008 (Invited Paper)

Presently, we run various prototype versions of the model in a rather ad-hoc fashion using Matlab. We are in the process of developing a more robust coding of the model processes in an object-oriented paradigm to improve the linkages with the outputs of geographical information systems and numerical weather prediction models. The mechanistic processes in the model are very general allowing us to simulate organism movement for many types of animals across the landscape with instantiation of individual species of interest.

Figure 1 gives an overall top-level view of the key components of the bird migration framework. For simplicity, details of the coupling of the bird movement routines to the spatially and temporally varying habitat and climate grids are shown schematically. The linkages may be developed off-line through preprocessing steps to build the necessary environmental and meteorological and climate layers or they can be linked in near-real time. Details of one of the methods we use to compute a temporally varying, en-route habitat suitability grid for potential stopover locations are given Sec 2.3 .

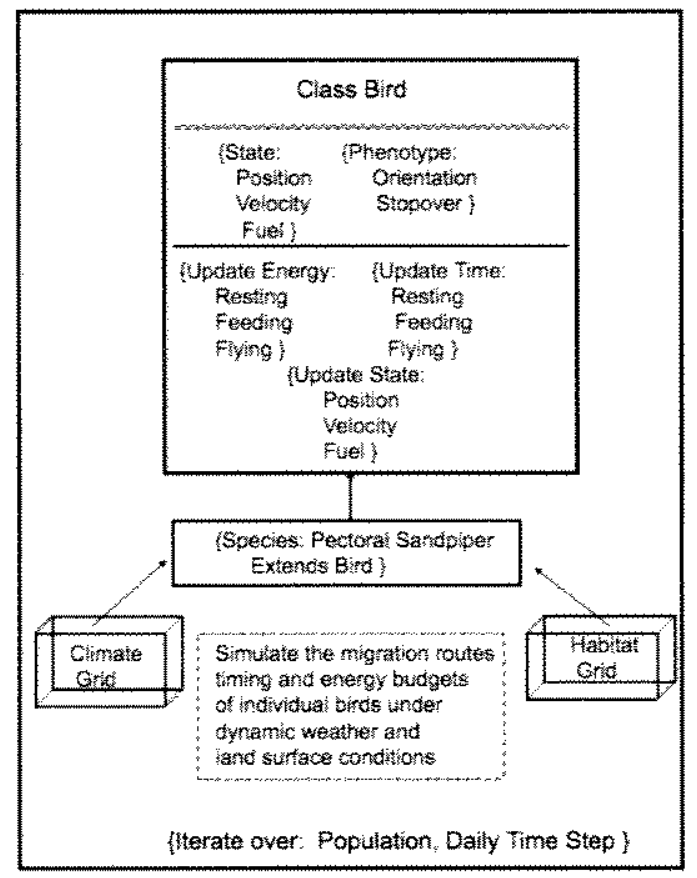

Figure 2. Overall top-level view of the key mechanistic model processes and class structure for individual -based bird migration model used in this study. The model integrates remote sensing data and near real-time or retrospective climate data to simulate the migratory routes, stopover behavior, and migration success of migratory birds.

The general bird class has both state and phenotype attributes. The state variables consist of the location, speed, direction, and fuel status of a bird. For computational efficiency, we also keep track on whether or not a bird is alive or dead or whether it has reached the breeding ground. Phenotype expression can be varied. For the simulations reported here, we selected the intrinsic behavior characteristics of endogenous flight direction (orientation) and stopover length.

The general bird class provides method stubs for updating the state variables by providing update methods for the bird time and energy budgets. Specific details are implemented in the Pectoral Sandpiper class that extends the class bird. Time spent resting, feeding, and flying and the corresponding energy gains or losses depend upon species morphology and physiology parameters that drive the flight model. The state variables are updated, taking into account the vector addition of the wind field components with local bird velocity.

The model instantiates a population of birds and iterates on a daily time-step during migration. The process is repeated for several generations. Each generation consists of instantiating a population of 10,000 birds by randomly drawing upon 
Proc SPIE ERS08 Proceedings, Vol 7104, Remote Sensing for Agriculture, Ecosystems, and Hydrology X, Cardiff, Wales, United Kingdom, September 15-19, 2008 (Invited Paper)

the distribution of successful phenotypic characteristics and random initial locations within the area of interest. Stability is generally reached within a few generations. The output of the model consists of individual migration routes, timing and energy budgets, and individual phenotypic characteristics. Information may be summarized over individual birds for populationmlevel distributions, and/or over geographic locations or time periods generating predictions to compare with available field or satellite observations of bird movements. For example, Figure 3 shows the results of summarizing the total number of birds passing each day through three latitudinal zones- 35 to 40 degrees, 40 to 45 degrees, and 45 to 50 degrees. Such bird "hydrographs" can be compared with similar data from the literature [3].

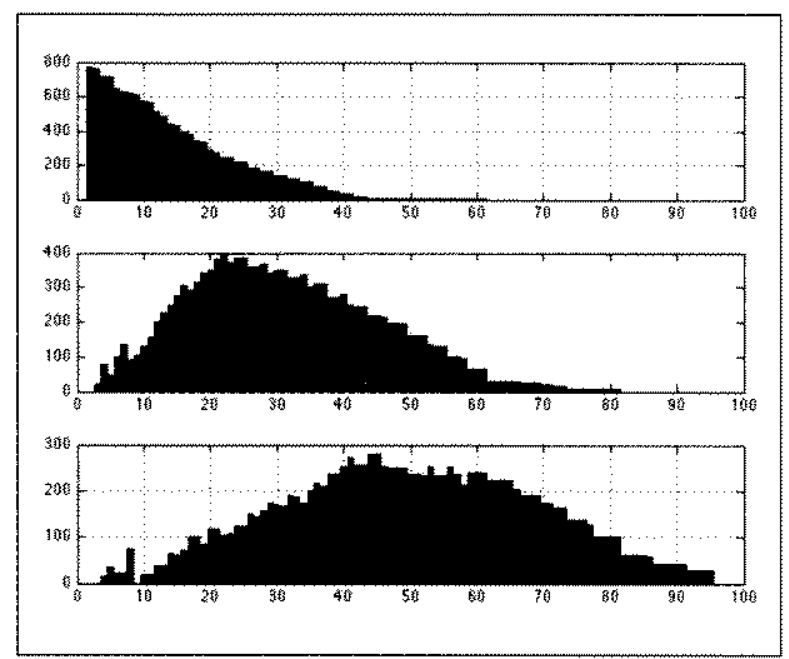

Figure 3. Bird "hydrograph" showing number of birds (vertical axis) passing through selected latitudinal zones each day of the spring migration period (horizontal axis). Counts are summed over varying 5 degree latitude bins. Top bar corresponds to latitude range 35 to $40 \mathrm{deg}$. Middle graph corresponds to 40 to 45 deg latitude range, and bottom graph to 45 to 50 deg latitude. General northward migration flow pattern is evident in the top to bottom plots.

\subsection{Estimating en-route spatio-temporal habitat suitability grid}

We used the MaxEnt (Maximum entropy) model of Phillips, et al [11] to predict the spatial distribution of potentially suitable environmental conditions for Pectoral Sandpipers during spring migration through North America. Here, we are using the distribution of suttable environmental conditions as a proxy for refueling potential. We acknowledge that some birds may occur in locations where they do not feed and refuel, in which case our maps may overestimate the distribution of suitable habitat. In these cases, the maps can be interpreted as a spatial representation of optimal conditions.

Maximum entropy is an ecological niche modeling approach that uses relationships between environmental variables and locations of known species occurrence to determine the conditions that are suitable for the species. Using these relationships, the model then predicts the distribution of those conditions throughout the study area of interest. Habitat suitability is modeled as a function of environmental variables that are thought to impact the species' survival and reproductive success (immediate or future). For migrating Pectoral Sandpipers, we considered factors that influence their ability to obtain food (e.g., number of days of frozen ground per month, precipitation) or physiological functioning (e.g., temperature) to be important variables.

Maximum entropy modeling estimates the probability distribution of suitable conditions that has maximum entropy across the study area within constraints that represent our incomplete knowledge. Two constraints are placed on the distribution: (1) it has to agree with all known information derived from the locations where the species has been recorded and (2) it cannot place any constraints on the distribution that are not supported by the available data.

Figure 4 shows the overall data flow and analysis steps in using MaxEnt to estimate habitat suitability. MaxEnt requires two data inputs: (1) a file containing bird occurrence records (training data) and (2) a set of environmental layers 
Proc SPIE ERS08 Proceedings, Vol 7104, Remote Sensing for Agriculture, Ecosystems, and Hydrology X, Cardiff, Wales, United Kingdom, September 15-19, 2008 (Invited Paper)

formatted as ASCIl raster grids. In addition, MaxEnt allows the user to input additional bird occurrence records for testing the model.

We acquired bird occurrence from three sources: United States Geological Survey Bird Banding Laboratory (BBL; http $/ /$ www pwrc.usgs gov/bbl/), Cornell Laboratory of Ornithology Avian Knowledge Network (AKN; http:/Www.avianknowledge.net/content/download), and the Global Biodiversity Information Facility (GBIF; http:/www.gbif.org/). Bird occurrence records were separated by month, and we retained $25 \%$ of the records from each month to validate, or test, our monthly models. We created separate models for April, May, and June representing the period of spring migration for Pectoral Sandpipers through the United States and Canada.

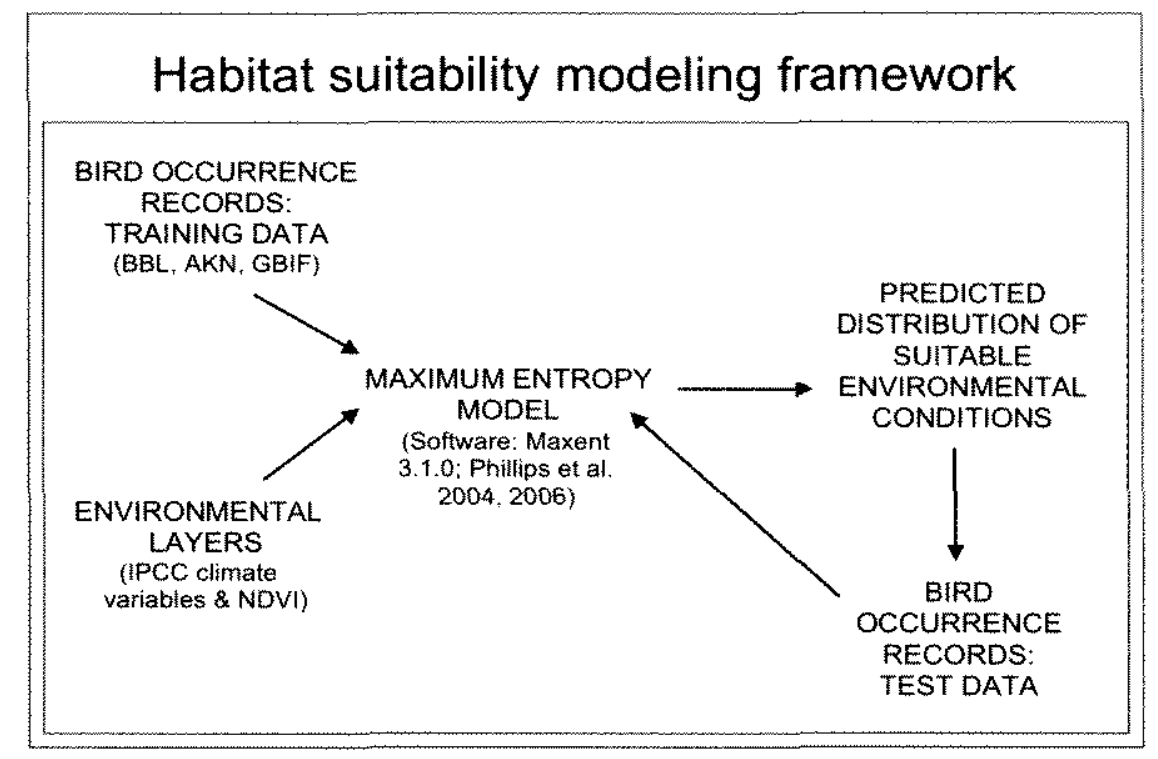

Figure 4. Data flow and analysis for estimating en-route habitat suitability distribution using Maximum Entropy method of Phillips, et al, (2006)

We used two sources for our environmental layers in our analysis. First, we used climate data from the Intergovernmental Panel on Climate Change (IPCC) available through the Data Distribution Center (htp/Wwwwipcedata org). These climate data represented 30-yr monthly averages over the period 1961-1990 with a 10 min resolution [10]. We used ten climate variables that we thought would directly or indirectly impact the survival, physiology, feeding rate, and timing of migration of Pectoral Sandpipers. These included precipitation, mean temperature, mean diurnal temperature range, relative humidity, ground frost (\# frost days), number of wet days, sunshine (\% of day length), and wind speed.

Secondly, we used the Normalized Difference Vegetation Index (NDVI; greenness index) calculated from Advanced Very High Resolution Radiometer (AVHRR) data [9] as a surrogate for soil moisture and vegetation growth. The original data sets had a resolution of $0.073 \mathrm{deg}$. We re-sampled data to $0.17 \mathrm{deg}$ (i.e., $10 \mathrm{~min}$ ) so that it was consistent with the IPCC climate layers. We averaged the 16-day composite data over the period 1981-1990, and then averaged again on a monthly basis to generate the monthly averages for April, May, and June.

Figure 5 shows an example predicted environmental suitability map derived for June. The colors indicate the strength of the prediction; green in various shades denotes a strong prediction of suitable environmental conditions for Pectoral Sandpipers, while shades of purple indicate more unsuitable conditions. 
Proc SPIE ERS08 Proceedings, Vol 7104, Remote Sensing for Agriculture, Ecosystems, and Hydrology X, Cardiff, Wales, United Kingdom, September 15-19, 2008 (Invited Paper)

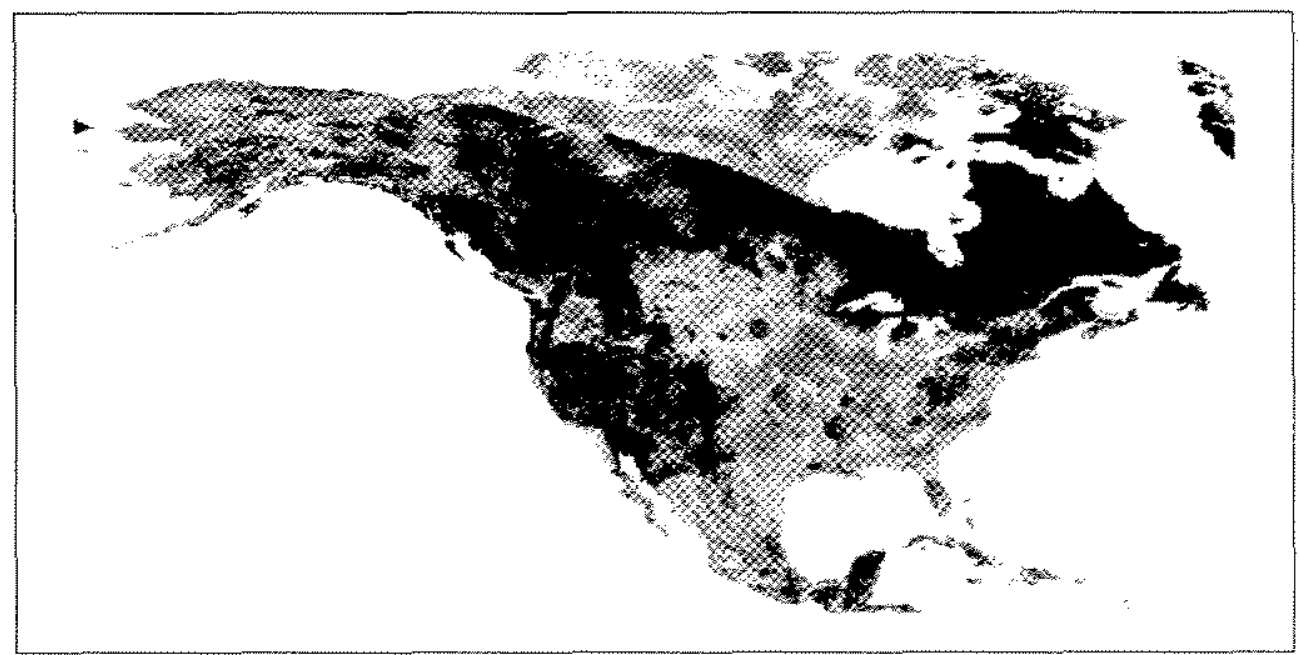

Figure. 5. Example June distribution of suitable environmental conditions derived from MaxEnt.using June monthly averaged environmental layers taken from the IPCC (New, et al. 2002) and NDVl (Tucker, et al. 2004). Green colors indicate areas of high potential.

\section{RESULTS AND DISCUSSION}

To run our simulations we used the body measurements and known physiology for pectoral sandpipers to estimate basal metobolic rate, energy expenditure during flight, refueling rates and maximum and minimum body fat loadings. A population of 10,000 birds was simulated with initial phenotypic variables of endogenous orientation and stopover length. Orientation directions were randomly sampled from the interval [320, to 350] in polar azimuth direction. Initial stopover lengths were randomly selected in the range $[1,20]$ days.

Starting latitudes were randomly selected from [30,35] degrees, and initial longitudes randomly selected from the range $[-94,-90]$ corresponding to the Texas coastal region. Mean bird speed was $15 \mathrm{~m} / \mathrm{s}$. We experimented with a random wind velocity component of $4 \mathrm{~m} / \mathrm{s}$.

Our simulation grid representing North America was divided into 10 minute by 10 minute latitude / longitude geographic grid cells, representing approximately 120,000 potential stopover locations. Migrating birds were able to move to any geographic coordinates according to their time and energy budget constraints and decision rules.

Any bird arriving at greater than or equal to a northern latitude of 65 deg with a minimum fat load and within the breeding time window was assumed successful. The phenotypic attributes of all successful migrants were then used to seed the next generation. Stability was achieved within a few generation cycles.

Figure 6 shows a sampling of the trajectories of the final generation (as also indicated in Figure 3 earlier). In general, the migration routes are consistent with known patterns [3]. The birds avoided flying over water bodies, e.g. the northeast Pacific and Hudson Bay) and avoided high elevation areas of low habitat suitability.

The equilibrium distribution of endogenous directions is shown in Figure 7 indicating less dispersion in the landscape then the initial range of potential starting directions. The distribution of stopover lengths is shown in Figure 8 . Stopover lengths were peaked near 10-14 days. The average optimum duration of about 13 days is consistent with observations [12]. 
Proc SPIE ERS08 Proceedings, Vol 7104, Remote Sensing for Agriculture, Ecosystems, and Hydrology X, Cardiff, Wales, United Kingdom, September 15-19, 2008 (Invited Paper)

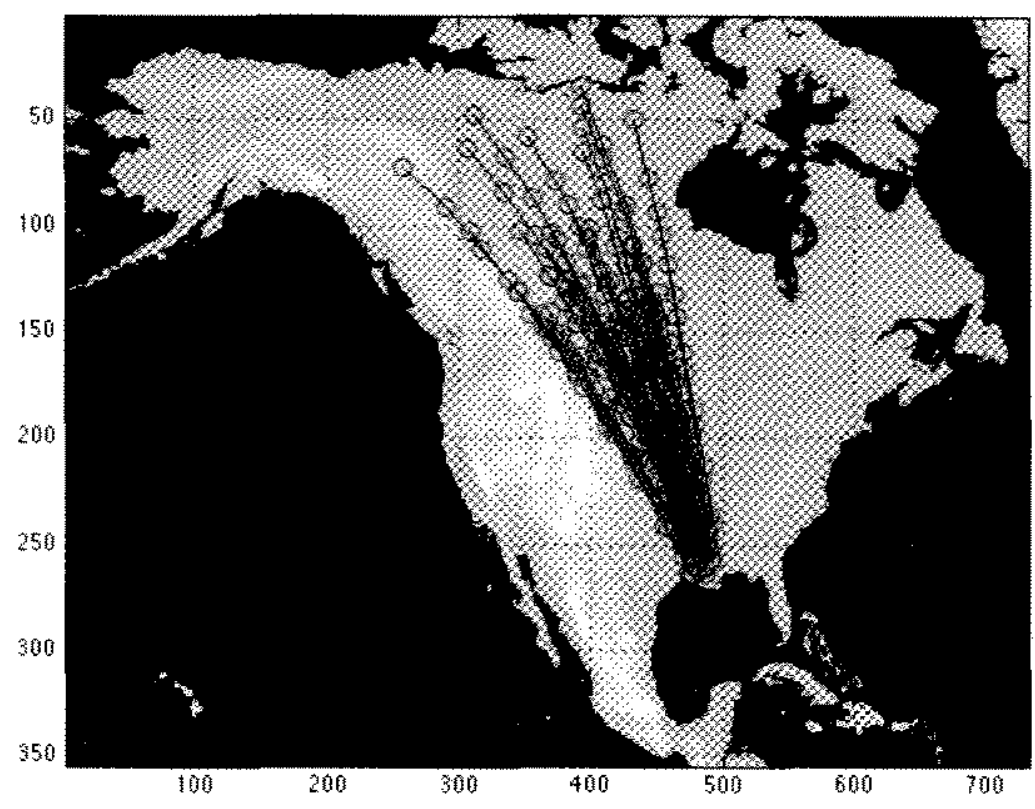

Figure. 6. Migration routes of a simulated population of 10,000 birds flown through a landscape of varying refueling or stopover potential. Subsample of trajectories is shown for graph clarity. Birds generally follow central North American flyway and avoid flying over high mountain elevations or large water bodies.

\section{CONCLUSIONS}

While the migration model presented here is a simplification of the complex phenomenon of bird migration, it generates consistent and plausible comparisons with observations [3] [12]. Alternatively, we can rum the model in calibration mode and tune model parameters by comparing and adjusting model patterns with those observed from survey data, stable isotope analyses [13], or tracking data [14].

By running the model for bird populations "flown" through alternative environmental scenarios, we can gain insight into the potential direction or relative magnitude of changes in migratory bird patterns with changes in climate and land surface conditions.

In the analyses presented here, we used a random wind component and simple vector addition to simulate net bird velocities as each bird was instantiated. The general model can assimilate either near-real time or retrospective meteorological data from numerical weather models. The logistics of coupling directly to the numerical weather prediction models can be a bit onerous. As part of a NASA funded Applied Information Sciences and Technology research effort awarded to Professor Liping Di at George Mason Lniversity entitled, "A general framework and system prototypes for self-adaptive Earth predictive systems-dynamically coupling sensor web with Earth system models," we are exploring more automated techniques for providing these linkages.

Finally, our use of remotely sensed land surface properties and climate data, together with bird location data to estimate candidate stopover locations, en-route habitat suitability, and potential fuel deposition rates remains an open challenge. While our work presented here is promising, there remain many questions. We were not able to directly use the probability distributions from MaxEnt without some judicious tweaking, particularly for the minimum probability stopovers. Whether this was from our use of the particular environmental layers, problems with the bird location data, or other factors remains to be further explored. 
Proc SPIE ERS08 Proceedings, Vol 7104, Remote Sensing for Agriculture, Ecosystems, and Hydrology X, Cardiff, Wales, United Kingdom, September 15-19, 2008 (Invited Paper)

Alternatively, we have experimented with a method similar to that employed by plant physiologists in estimating stomatal conductance as a function of various abiotic factors. In that work, we related potential fuel deposition rates or stopover probabilities more directly to abiotic and biotic, spatially and temporally varying environmental layers. So far, the results look very encouraging.

\section{ACKNOWLEDGEMENTS}

Our research is supported under a NASA Interdisciplinary Science research proposal entitled, "Forecasting the effects of wetland loss and inter-annual variability on the fitness of migratory bird species," and, in part, by a NASA Applied Information Sciences and Technology proposal entitled, "A general framework and system prototypes for self wadaptive Earth predictive systems-dynamically coupling sensor web with Earth System models." We appreciate helpful discussions with Dr. Susan Skagan and Adrian Farmer with the USGS Fort Collins Science Center and with Mr. Russell Oates, Chief Migratory Bird Management, U.S. Fish and Wildlife Service-Alaska.

\section{REFERENCES}

11] Winter, T. C., "The vulnerability of wetlands to climate change: a hydrologic landscape perspective," J. of the American Water Resources Association, 36:305-311 (2000)

[2] Brown, S., C. Hickey, B. Harrington, and R. Gill, eds, [The U.S. Shorebird Conservation Plan, $2^{\text {nd }}$ Ed.] Manomet, MA, Manomet Center for Conservation Sciences (2001)

[3] Skagen, S.K., "Migration stopovers and the conservation of Arctic-breeding Calidridine Sandpipers," AUK 123:313 322(2006)

[4] Smith, James A. and Jill L. Deppe, "Simulating the effects of wetland loss and inter-annual variability on the fitness of migratory bird species," Proc. IEEE Geosciences and Remote Sensing Symposium, Boston MA, November 2008 (In press)

[5] Altobelli, Alfredo, Tatsiana Hubina, Alberto Sisto, and Stefano Sponza, "Effect of abiotic and biotic factors on the abundance of waterbirds in Grado-Marano Lagoon (ltaly)," SPIE ERS08 7104 (2008)

[6] Schnase, J. L., J. Cushing, and J.A. Smith, Biodiversity and ecosystem informatics. J. Intell Inf. Syst., DOI $10.1007 / \mathrm{s} 108444-006-002-7(2007)$

[7] Smith, J.A. and J. L. Deppe, "Simulating bird migration using satellites and biophysics," Proc. Of the IASTED Symposium on Envitonmental Modeling and Simulation 509, 6-11 (2007)

[8] Deppe, J.L., K. Wessels, and J.A. Smith, "Alaska at the crossroads of migration: space-based onithology," Alaska Park Science, 6:53-58 (2007)

[9] Tucker, CJ., J.E. Pinzon, and M.E. Brown, "Global Inventory Modeling and Mapping Studies, NA04apr15b.n1 1Vlg,2.0". Global Land Cover Facility, University of Maryland, College Park, Maryland, 04/15/1994 (2004)

[16] New, M., D. Lister, M. Hulme, and I. Makin, "A high-resolution data set of surface climate over global land areas," Climate Research, 21:1 25 (2002)

[1] S.J. Phillips, R.P. Anderson, and R.E. Schapire, "Maximum entropy modeling of species geographic distributions," Ecological Modeling, 190:231-259 (2006)

(12) Farmer, A.H. and John Weins, "Optimal migration schedules depend on the landscape and the physical environment: a dynamic modeling view," J. Avian biology, 20:405-415 (1998)

[13] Wikelski, M., R.W. Kays, N.J. Kasdin, K. Thorup, J.A. Smith, and G.W. Swenson, Jr. "Going wild: what a global small-animal tracking system could do for experimental biologists," Journal of Experimental Biology, 210: 181-186 $(2007)$

[14] Hobson, Keith A and Leonard I. Wassenaar, eds. Tracking animal migration with stable isotopes. Elsevier Science and Technology Books ISBN-13:9780123738677, 144p (2008). 
Proc SPIE ERS08 Proceedings, Vol 7104, Remote Sensing for Agriculture, Ecosystems, and Hydrology X, Cardiff, Wales, United Kingdom, September 15-19, 2008 (Invited Paper)

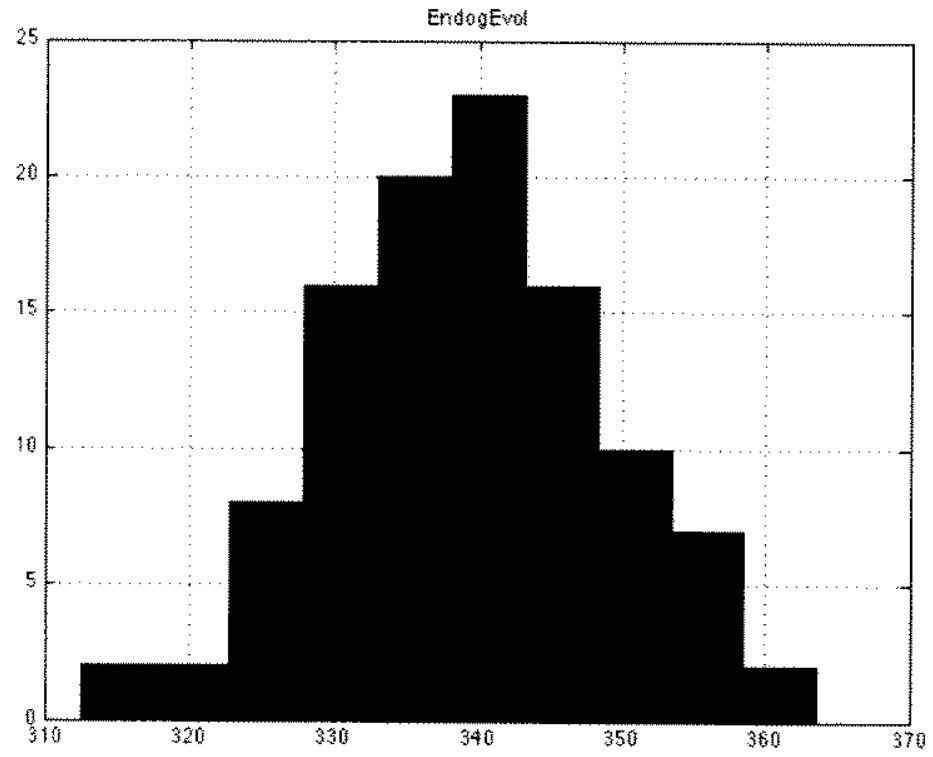

Figure. 7. Distribution of intrinsic phenotypic endogenous direction attributes for migratory bird population reachign the breeding ground. As birds evolved their behavior to adapt to the varying environmental conditions, they became less dispersed in the landscape and avoided low fuel deposition areas at high elevations or over large water bodies.

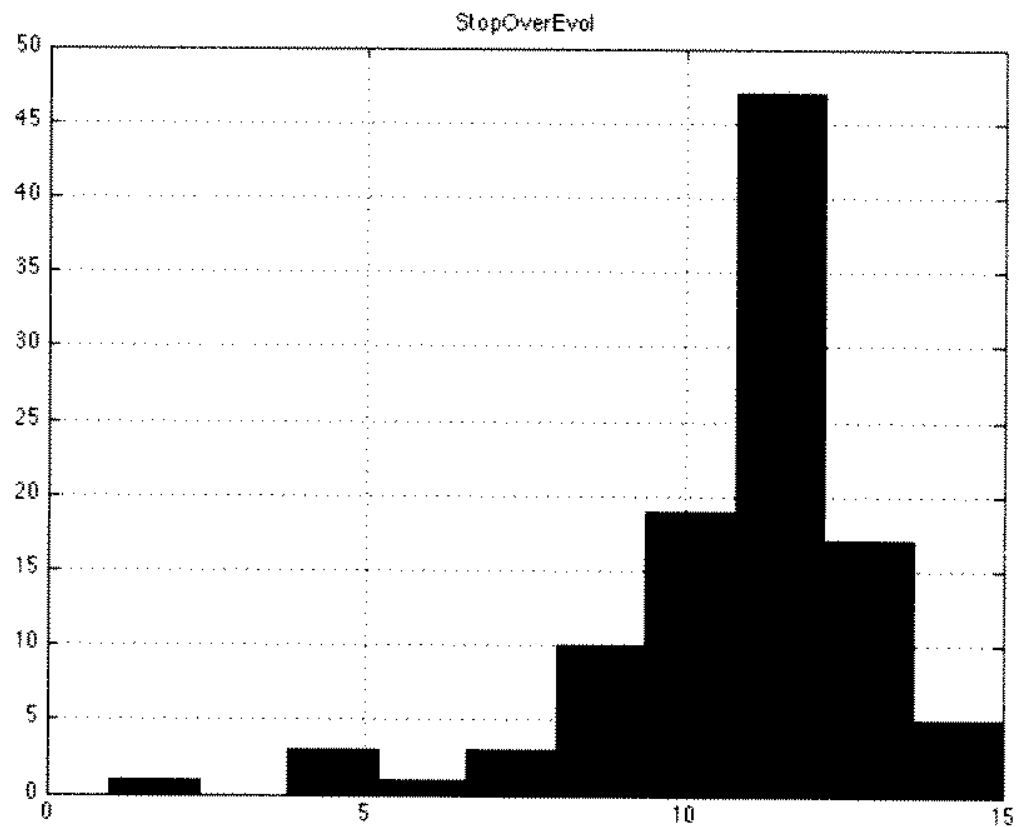

Figure. 8. Distribution of stopover duration length for migratory bird population successfully reaching the breeding grounds. Mean stopover length of 12 to 13 days is consistent with observed trends. 